\title{
Primary CNS Malignant Melanoma Associated With a Contralateral Nevus of Ota in an African-American Female: Unique Case Report
}

\author{
Bharat Guthikonda ${ }^{1}$, Osama Ahmed ${ }^{1}$, Linda J Buckleair ${ }^{2}$, J Clay Goodman ${ }^{3}$, \\ Suzanne Z Powell ${ }^{4}$ \& Daniel Yoshor ${ }^{5}$ \\ ${ }^{1}$ Department of Neurosurgery, Louisiana State University Health Sciences Center, Shreveport, LA, USA \\ ${ }^{2}$ Department of Pathology, Baylor University Medical Center, Dallas, TX, USA \\ ${ }^{3}$ Department of Pathology, Baylor College of Medicine, Houston, TX, USA \\ ${ }^{4}$ Department of Pathology, The Methodist Hospital, Houston, TX, USA \\ ${ }^{5}$ Department of Neurosurgery, Baylor College of Medicine, Houston, TX, USA \\ Correspondence: Osama Ahmed, MD, Louisiana State University Health Sciences Center, Department of \\ Neurosurgery, 1501 Kings Highway, Shreveport, LA 71103, USA. Tel: 1-318-675-6404; Fax: 1-318-675-6867. \\ E-mail: oahmed@1suhsc.edu
}

Received: December 23, 2014 Accepted: Febtuary 3, 2015 Online Published: March 24, 2015

doi:10.5539/cco.v4n1p39 URL: http://dx.doi.org/10.5539/cco.v4n1p39

\begin{abstract}
Background: Primary CNS melanoma is a rare neoplasm of the brain. Nevus of Ota is an area of macular, melanocytic pigmentation of the sclera and skin around the eye. Association between intracranial melanocytic neoplasms and Nevus of Ota has previously been described in case reports. The two lesions tend to occur ipsilaterally and in Caucasian patients. We present the first documented case of a primary malignant CNS melanoma contralateral to a Nevus of Ota in an African-American patient.
\end{abstract}

Case Discussion: A 32-year-old female presented with intermittent numbness involving the left side of her face, arm, and leg. General physical exam revealed a large area of hyperpigmentation involving the left side of her face, especially around the eye and upper cheek, consistent with Nevus of Ota. Neuroimaging revealed a hemorrhagic intraparenchymal mass in the right frontotemporal region. Surgical resection of the lesion was performed, and pathology was consistent with primary CNS malignant melanoma.

Conclusion: Primary CNS melanocytic neoplasms and Nevus of Ota share a similar embryological origin. Thus, the associated occurrence of the two is not surprising. We present a unique case of primary CNS malignant melanoma associated with a contralateral Nevus of Ota in an African-American patient. Primary CNS melanoma should be considered in the differential diagnosis of any patient with a Nevus of Ota who is found to have an intracranial mass.

Keywords: central nervous system, malignant, melanoma, nevus of ota
Abbreviations:
CNS-central nervous system
CT-computed tomography
MRI-magnetic resonance imaging
HMB-human melanoma black

\section{Introduction}

Primary CNS (central nervous system) melanoma is a relatively rare neoplasm of the brain, accounting for $1 \%$ of all melanoma cases. The peak incidence of primary CNS melanoma occurs in the fourth decade (Brat, 1999). It usually occurs in Caucasians, with females more frequently affected than males (Kramer, 1992). When these neoplasms do occur, they are noted for their propensity to hemorrhage.

Nevus of Ota, also known as oculodermal melanocytosis or nevus fusca-ceruleus ophthalmomaxillaris, is an extensive patch-like area of dermal melanocytic pigmentation of the sclera and skin around the eye (Hino, 2005; 
Ota, 1939). It is a manifestation of the same melanocytic proliferation that leads to primary CNS melanocytic lesions. It is thought to occur due to abnormal migration of melanocytes, which prematurely arrest in the dermis rather than at the usual dermal-epidermal junction (Hino, 2005). Nevus of Ota is an extremely rare lesion, but when it does occur, it most commonly affects the Asian population with a strong female propensity. It is a lesion with a characteristic gross diagnostic appearance. Therefore, skin biopsy is often not needed for diagnosis, though it is sometimes performed for academic interest.

There is a known association between melanocytic intracranial lesions and the Nevus of Ota (Arunkumar, 2001; Balmaceda, 1983; Botticelli, 1983; Clarke, 1998; Enriquez, 1973; Gomez-Gonzalez, 1965; Hartmann, 1989; Hino, 2005; Horsey, 1980; Kabuto, 1980; Kojima, 1959; Piercecchi-Marti, 2002; Rahimi-Movaghar, 2003; Rivers, 2001; Rutten, 2005; Sagar, 1983; Sang, 1977/; Theunissen, 1993; Willis, 1967). These reports have most commonly consisted of intracranial melanoma or melanocytoma ipsilateral to the Nevus of Ota in Caucasian patients. We present a unique case of primary malignant CNS melanoma located contralateral to a Nevus of Ota in an African-American female.

\section{Case Report}

A 32-year-old African-American female initially presented with intermittent episodes of left-sided numbness involving her face, arm, and leg that were associated with mild head "pressure." She had no associated weakness, nausea, vomiting, vision change, or bowel/bladder incontinence. She also had no generalized convulsions. These episodes would last several seconds and would resolve spontaneously. When she suffered her third such episode, she sought medical attention.

The patient had no significant past medical or surgical history. Specifically, she had no history of renal disease or hypertension. The patient worked as an attorney and did not drink alcohol, smoke cigarettes, or use any illicit drugs. Specifically, she did not have any history of cocaine or amphetamine use. Her family history revealed no evidence of neurological disease.

General physical exam revealed the presence of a large area of hyperpigmentation involving the left side of her face, especially around the eye and upper cheek (Figure 1).

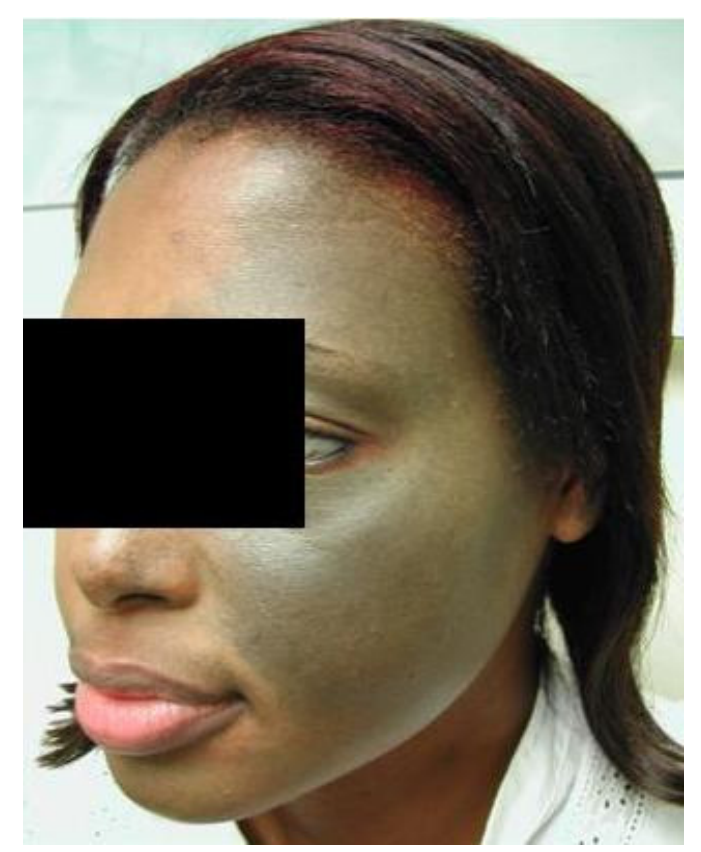

Figure 1. Patient with left-sided macular hyperpigmented lesion involving the distribution of the first (V1) and second (V2) branches of the trigeminal nerve, consistent with Nevus of Ota. Note focal scleral involvement

The lesion was a heterogeneous, macular region that was black in color. It was significantly darker than her normal skin pigment. This lesion has been present since birth and has not shown any significant change in size or character in her adult life. Her neurological exam at presentation was normal.

She underwent a CT (computed tomography) scan of the brain with and without contrast that revealed a right 
fronto-temporal intracranial hemorrhage $(2.0 \mathrm{~cm} \times 2.5 \mathrm{~cm} \times 2.0 \mathrm{~cm})$ with a rim of surrounding vasogenic edema (Figure 2).

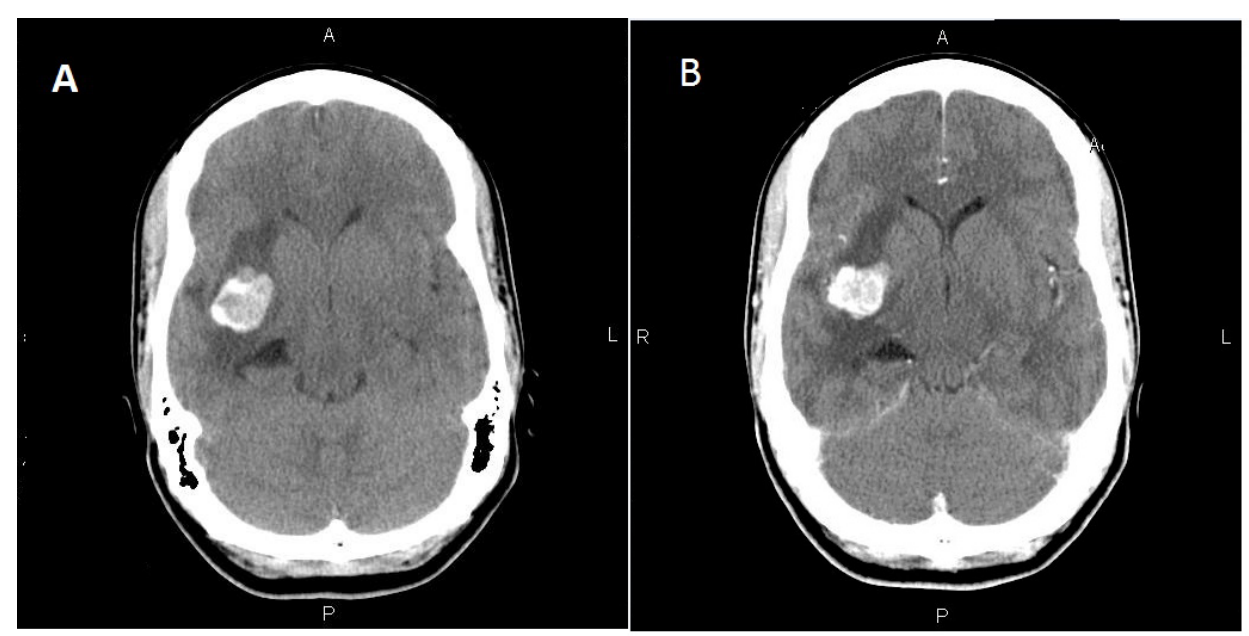

Figure 2. CT images from a 32-year-old woman with numbness of the left arm, leg and left side of face. Axial non-contrast (A) and contrast-enhanced (B)

CT images show a hemorrhagic intraparenchymal mass in the right frontal and temporal lobes, surrounding the sylvian fissure. Note vasogenic edema surrounding the lesion.

Given the hemorrhagic nature of the lesion and unremarkable past medical history of the patient, the decision was made to perform a diagnostic 4-vessel cerebral angiogram to exclude an underlying arteriovenous malformation or aneurysm. No vascular abnormalities were found. The patient remained asymptomatic during her hospitalization and was neurologically intact on examination. The decision was made to allow the patient to recover from the intracranial hemorrhage with planned repeat imaging two months later. She was also placed on phenytoin for seizure prophylaxis.

MRI (magnetic resonance imaging) of the brain with and without gadolinium (Figure 3) was performed two months later and revealed that the hemorrhagic lesion had actually expanded in size and still contained a significant amount of surrounding edema. 

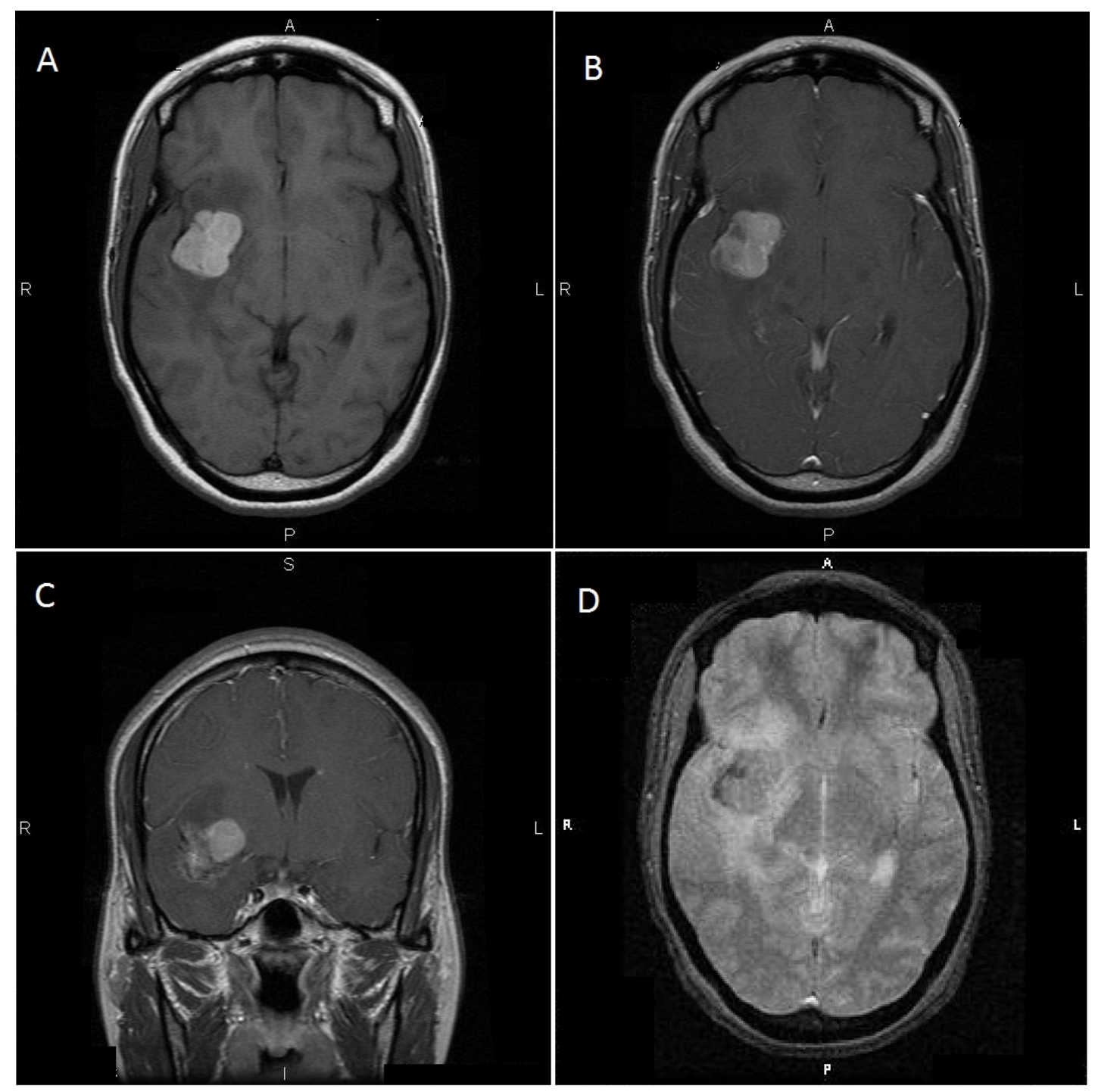

Figure 3. MRI images from same patient

Axial T1-weighted image (A) reveals the right frontotemporal hemorrhagic mass. Gadolinium-enhanced axial (B) and coronal (C) views of the large, right frontotemporal hemorrhagic mass with surrounding edema. Axial gradient-echo image (D) demonstrates hemorrhagic nature of lesion and surrounding vasogenic edema. Gadolinium-enhanced axial (A, B) and coronal (C) T1-weighted images reveal gross total resection of the lesion.

This further confirmed the suspicion that there was a tumor associated with this hemorrhagic area. The decision was made to proceed with surgical resection of the lesion to both relieve the mass effect and to obtain a definitive diagnosis.

A right frontotemporal craniotomy was performed using frameless stereotactic navigation. Once the right frontal and temporal lobes were exposed, we noted that there were diffuse punctuate foci of black hyperpigmentation throughout the subarachnoid space. This was noted in all the sulcal spaces exposed. The mass was approached by a transsylvian corridor using the microscope. The tumor was black and moderately firm on the outer surface. There were areas of hemorrhage within the mass, but separate areas of abnormal tissue without hemorrhage were also identified and resected. Gross total resection was performed, and the mass was sent for pathological identification.

Cytologic touch imprint of the tumor at the time of frozen section consisted almost entirely of fine, black melanotic pigment with occasional clusters of malignant cells. Frozen and permanent sections demonstrated a densely pigmented tumor with necrosis (Figure 4). 


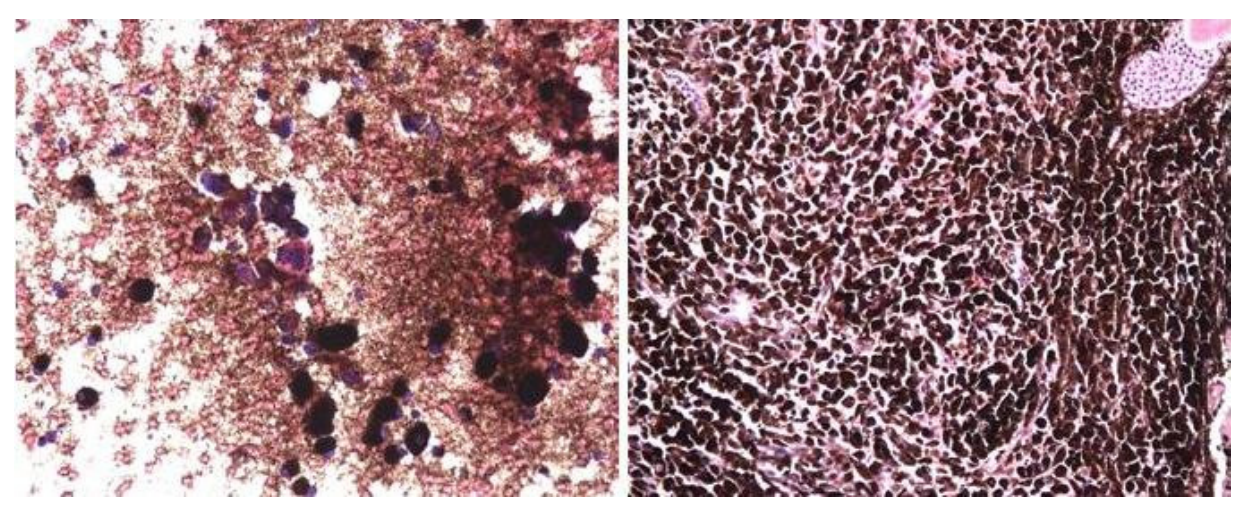

Figure 4. A - Intraoperative cytological imprint (touch prep) showing dense pigmentation and atypical, binucleate cells (10x). B - Low power view of frozen section, confirming dense pigmentation and hypercellularity $(10 \mathrm{x})$

Where unobscured by pigment, the nuclei were highly pleomorphic, with high nuclear/cytoplasmic ratios and numerous prominent nucleoli, many of which were eosinophilic. Few mitotic figures were noted. Bleached sections allowed better appreciation of the hypercellularity, nuclear pleomorphism with prominent eosinophilic nucleoli, and necrosis (Figure 5).
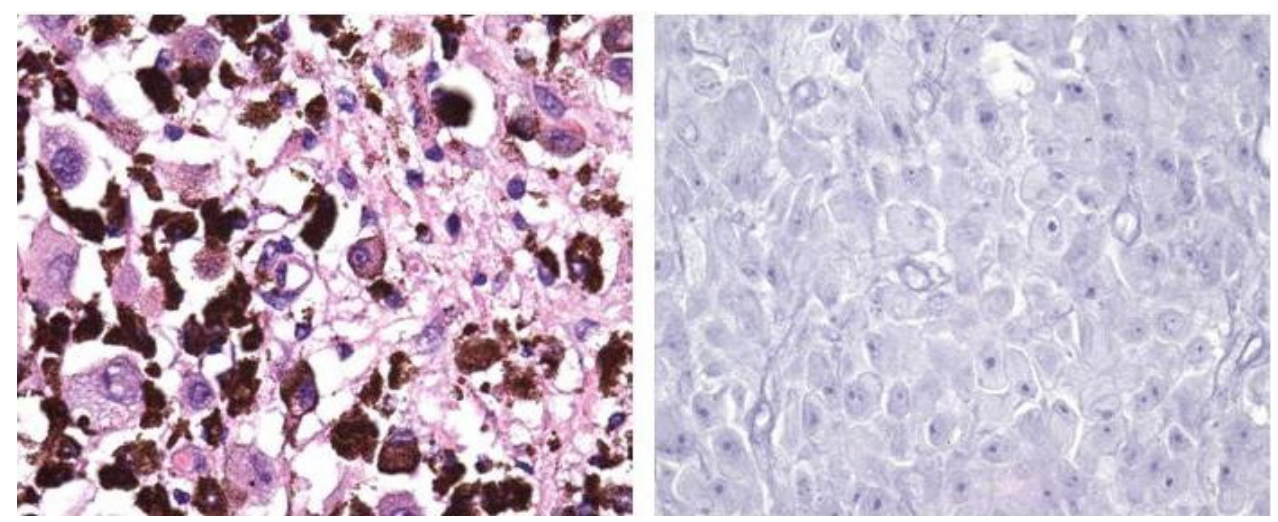

Figure 5. A - Higher power view of permanent section. Note marked cellular atypia. (40x). B - Bleached section better demonstrating malignant cells with large nucleoli (40x)

Focal perivascular infiltration of tumor was noted in adjacent neuropil. Pathological diagnosis was consistent with primary central nervous system malignant melanoma. The patient is undergoing adjuvant radiation therapy.

Given the association of Nevus of Ota with pigmented ocular lesions (typically affecting the sclera, cornea, and conjunctiva), a complete ophthalmological examination was performed. No abnormal findings were found.

\section{Discussion}

Nevus of Ota, also referred to as oculodermal melanocytosis or nevus fuscoceruleus opthalmomaxillaris, is a hyperpigmented lesion usually involving the distribution of the first and second branches of the trigeminal nerve. Thus, it commonly affects the skin around the eye and on the cheek. It was first described by Hulke in 1861 in association with malignant melanoma of the sclera. In 1939, Ota described a series of 26 cases in Japan (Ota, 1939). It occurs either congenitally $(60 \%)$ or has onset at puberty $(40 \%)$. It is usually a unilateral, macular lesion that varies in color from blue to black with heterogeneity often seen within the same lesion. It typically gets darker during adolescence and persists into adulthood. It most commonly affects the Asian population with a strong female propensity (4:1). Histopathologically, it is a patch-like area of dermal melanocytic pigmentation. An association exists with pigmented lesions involving the eye, especially the sclera, episclera, and conjunctiva. These ocular manifestations are most commonly noted in African-Americans and Asians and are overall seen in about $50-60 \%$ of cases. Nevus of Ota has also been reported in association with Takayasu's arteritis (Reinke, 1974), Klippel-Trenaunay syndrome (Furukawa, 1970), and Type 1 neurofibromatosis (Clarke, 1998). Given its 
unique gross appearance and distribution, biopsy is rarely indicated for diagnostic purposes.

Nevus of Ota and intracranial melanocytic lesions share a similar embryologic origin. Melanoblasts develop from neural crest origin and are seen as early as the $10^{\text {th }}$ week of fetal life. They differentiate into melanocytes, which are fusiform elements with the ability to produce melanin. These normally migrate to skin, meninges, ocular structures, and the inner ear (Botticelli, 1983). In the skin, melanocytes are normally found at the dermal/epidermal junction. Occasionally, due to an error in migration, some melanocytes become arrested in the dermis. Due to this migration anomaly, a variety of dermatopathology may occur, including a Mongolion spot, cellular blue nevus, and a Nevus of Ota.

In the CNS, melanocytes are specifically located in the pia, which is the neural crest component of the meninges. They are diffusely distributed in the CNS, but do have a higher concentration in the ventral medulla oblongata and upper cervical spinal cord. Neoplastic transformation of these melanocytes can lead to a spectrum of primary CNS melanoctyic tumors. These range from melanocytoma (which are benign lesions with an excellent prognosis) to malignant melanoma (with aggressive histologic features and a poor prognosis) (Rahimi-Movaghar, 2003). These are extremely rare lesions with less than 200 reported cases.

The neuroimaging appearance of melanoma is quite variable, depending on the melanin content and also on the presence of hemorrhage, which is often seen with both primary and metastatic malignant melanoma. On non-enhanced CT scan, melanomas are often hyperdense, and they enhance fairly homogenously with intravenous contrast. They are typically hyperintense on T1 weighted MRI and hypointense on T2 weighted MRI. Angiography is rarely performed, but does reveal an occasional blush due to pial blood supply.

Light microscopic findings of melanoma include sheets of polygonal and elongated cells with round or oval hyperchromic nuclei and eosinophilic cytoplasm that contains brown, granular pigment. Cells are often fusiform in shape and are sometimes arranged in lobules. Similar to other neuro-oncological disease, frequent mitoses, hypercellularity, nuclear pleomorphism, and perivascular infiltration are characteristic of malignant neoplasms that would not be seen in a melanocytoma. Electron microscopy, though rarely performed, reveals a lack of tight junctions and melanosomes that are in various stages of development. There are also no desmosomes or interdigitating cytoplasmic processes, in contrast to meningioma.

Immunohistochemistry evaluation is often performed if the melanotic lesion is dural based in order to distinguish it from meningioma. Melanomas stain positively for HMB-45 (anti-melanosomal antibody), S-100, and vimentin and are reactive with epithelial membrane antigen (which is often positive in meningioma).

An association between primary CNS melanoma and Nevus of Ota has been demonstrated in the literature. Fourteen cases have been previously reported (Table 1).

Table 1.Primary CNS melanomas occurring with Nevus of Ota reported in the literature

\begin{tabular}{lllll}
\hline Author & Year & Age & Sex & Relationship to facial lesion \\
\hline Willis & 1948 & 46 & F & Ipsilateral \\
Kojima, et al & 1959 & 37 & M & Ipsilateral \\
Gomez-Gonzalez, et al & 1965 & 66 & F & Ipsilateral \\
Enriquez, et al & 1973 & 43 & M & Midline \\
Sang, et al & 1977 & 58 & M & Contralateral \\
Horsey, et al & 1980 & 37 & F & Ipsilateral \\
Sagar, et al & 1983 & 23 & F & Ipsilateral \\
Kabuto, et al & 1988 & 77 & M & Ipsilateral \\
Hartmann, et al & 1989 & 41 & F & Ipsilateral \\
Balmaceda, et al & 1993 & 43 & F & Ipsilateral \\
Same & 1993 & 52 & F & Contralateral \\
Theunissen, et al & 1993 & 30 & F & Ipsilateral \\
Rutten, et al & 2005 & 37 & F & Ipsilateral \\
\hline
\end{tabular}




\begin{tabular}{lllll}
\hline Hino, et al & 2005 & 75 & F & Ipsilateral \\
Guthikonda, et al & 2007 & 32 & F & Contralateral
\end{tabular}

This is not surprising given the similar pathophysiology of the two lesions. The majority of these cases (58\%) have involved lesions that were dural-based. Our case is especially interesting in two separate regards. First, the intracranial melanoma was located contralateral to the Nevus of Ota. This has only been reported twice before in the literature. Second, our patient is of African-American descent. The simultaneous occurrence of primary CNS melanoma and contralateral Nevus of Ota has not previously been documented in an African-American patient. Despite the fact that these lesions are both rare, one should consider primary CNS melanoma in the differential diagnosis in a patient with Nevus of Ota who presents with an intracranial mass lesion.

\section{Conclusion}

Primary CNS melanoma and a Nevus of Ota have been rarely reported to occur simultaneously. We present a unique example of this occurrence in an African-American female. It is made more unique because the intracranial lesion is contralateral to the Nevus of Ota. Primary CNS melanoma should be considered in the differential diagnosis in a patient with Nevus of Ota and an intracranial mass.

\section{References}

Arunkumar, M. J., Ranjan, A., Jacob, M., \& Rajshekhar, V. (2001). Neurocutaneous melanosis: a case of primary intracranial melanoma with metastasis. Clinical oncology (Royal College of Radiologists (Great Britain)), $13,52-54$.

Balmaceda, C. M., Fetell, M. R., O'Brien, J. L., \& Housepian, E. H. (1993). Nevus of Ota and leptomeningeal melanocytic lesions. Neurology, 43, 381-386. http://dx.doi.org/10.1212/WNL.43.2.381

Botticelli, A. R., Villani, M., Angiari, P., Peserico, L. (1983). Meningeal melanocytoma of Meckel's cave associated with ipsilateral Ota's nevus. Cancer, 51, 2304-2310. http://dx.doi.org/10.1002/1097-0142(19830615)51:12<2304::AID-CNCR2820511223>3.0.CO;2-U

Brat, D. J., Giannini, C., Scheithauer, B. W., \& Burger, P. C. (1999). Primary melanocytic neoplasms of the

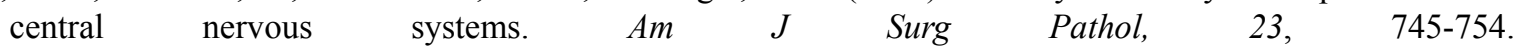
http://dx.doi.org/10.1097/00000478-199907000-00001

Clarke, D. B., Leblanc, R., Bertrand, G., Quartey, G. R., \& Snipes, G. J. (1998). Meningeal melanocytoma. Report of a case and a historical comparison. Journal of Neurosurgery, 88, 116-121. http://dx.doi.org/10.3171/jns.1998.88.1.0116

Enriquez, R., Egbert, B., \& Bullock, J. (1973). Primary malignant melanoma of central nervous system. Pineal involvement in a patient with nevus of ota and multiple pigmented skin nevi. Archives of Pathology, 95, 392-395.

Furukawa, T., Igata, A., Toyokura, Y., \& Ikeda, S. (1970). Sturge-Weber and Klippel-Trenaunay syndrome with nevus of Ota and Ito. Archives of dermatology, 102(6), 640-645. http://dx.doi.org/10.1001/archderm.1970.04000120058010

Gomez-Gonzalez, J. R. A., \& Rodriguez, A. (1965). Melanosis oculodermica, melanoblastosis leptomeningea y melanoma intracerebral primario. Universitas Medica, 47-52.

Hartmann, L. C., Oliver, G. F., Winkelmann, R. K., Colby, T. V., Sundt, T. M., \& O'neill, B. P. (1989). Blue nevus and nevus of Ota associated with dural melanoma. Cancer, 64(1), 182-186. http://dx.doi.org/10.1002/1097-0142(19890701)64:1<182::AID-CNCR2820640131>3.0.CO;2-P

Hidano, A., Kajima, H., Ikeda, S., Miyasato, H., \& Niimura, M. (1967). Natural history of nevus of Ota. Archives of dermatology, 95(2), 187-195. http://dx.doi.org/10.1001/archderm.1967.01600320043006

Hino, K., Nagane, M., Fujioka, Y., \& Shiokawa, Y. (2005). Meningeal melanocytoma associated with ipsilateral nevus of Ota presenting as intracerebral hemorrhage: case report. Neurosurgery 56, E1376.

Horsey, W. J., Bilbao, J. M., Nethercott, J., Myers, R., \& Hoffman, H. J. (1980). University of Toronto neurosurgical rounds no. 2. Oculodermal melanosis (naevus of Ota) complicated by multiple intracranial tumors. The Canadian Journal of Neurological Sciences, 7, 101-107.

Kabuto, M., Hayashi, M., Kawano, H. et al. (1980). [Primary intracranial malignant melanoma associated with nevus of Ota: a case report]. No shinkei geka 16, 875-880. 
Kojima, K. N. C., Honda, A., \& Miyagawa, C. (1959). A melanoma of the optic chiasm occurred in a case of Ota's naevus and melanosis bulbi. Jpn J Clin Ophthalmol, 502-504.

Kramer, I. R. P. J., \& Shear, M. (1992). Histological typing of odontogenic tumors. Berlin: Springer Verlag, 193-195, 1992. http://dx.doi.org/10.1007/978-3-662-02858-2

Ota, M. (1939). Nevus fusco-caeruleus ophthalmomaxillaris. Jpn J Dermatol, 369-372.

Piercecchi-Marti, M. D., Mohamed, H., Liprandi, A., Gambarelli, D., Grisoli, F., \& Pellissier, J. F. (2002). Intracranial meningeal melanocytoma associated with ipsilateral nevus of Ota: case report. Journal of neurosurgery, 96(3), 619-623. http://dx.doi.org/10.3171/jns.2002.96.3.0619

Rahimi-Movaghar, V., \& Karimi, M. (2003). Meningeal melanocytoma of the brain and oculodermal melanocytosis (nevus of Ota): case report and literature review. Surgical neurology, 59(3), 200-210. http://dx.doi.org/10.1016/S0090-3019(02)01052-2

Reinke, R. T., Haber, K., \& Josselson, A. (1974). Ota nevus, multiple hemangiomas, and Takayasu arteritis. Archives of dermatology, 110(3), 447-450. http://dx.doi.org/10.1001/archderm.1974.01630090081022

Rivers, J. K., Bhayana, S., \& Martinka, M. (2001). Dural melanoma associated with ocular melanosis and multiple blue nevi. Journal of Cutaneous Medicine and Surgery: Incorporating Medical and Surgical Dermatology, 5(5), 381-385. http://dx.doi.org/10.1007/s10227-001-0004-2

Rutten, I., Bolle, S., Kaschten, B., Stevenaert, A., Deneufbourg, J. M., \& Deprez, M. (2005). Recurrent intracranial melanocytoma associated with a nevus of Ota. Acta neurochirurgica, 147(3), 313-315. http://dx.doi.org/10.1007/s00701-004-0457-3

Sagar, H. J., Ilgren, E. B., \& Adams, C. B. (1983). Nevus of Ota associated with meningeal melanosis and intracranial melanoma: Case report. Journal of neurosurgery, 58(2), 280-283. http://dx.doi.org/10.3171/jns.1983.58.2.0280

Sang, D. N., Albert, D. M., Sober, A. J., \& McMeekin, T. O. (1977). Nevus of Ota with contralateral cerebral $\begin{array}{llll}\text { melanoma. } & \text { Archives }\end{array}$ http://dx.doi.org/10.1001/archopht.1977.04450100122017

Theunissen, P., Spincemaille, G., Pannebakker, M., \& Lambers, J. (1993). Meningeal melanoma associated with nevus of Ota: case report and review. Clinical Neuropathology, 12, 125-129.

Willis, R. (1967). Pathology of Tumors. London: Butterworth (pp. 930-931).

\section{Copyrights}

Copyright for this article is retained by the author(s), with first publication rights granted to the journal.

This is an open-access article distributed under the terms and conditions of the Creative Commons Attribution license (http://creativecommons.org/licenses/by/3.0/). 